\title{
New Insights on the Local ISM from EUV Observations
}

\author{
Stuart Bowyer \\ Department of Astronomy and Space Sciences Laboratory University of California \\ at Berkeley, California 94720-7450, USA
}

\begin{abstract}
New results from $E U V E$ are reviewed, with emphasis on the ionization state of the ISM and the question of thermal pressure balance between warm clouds and the hot intercloud medium. A description of what may be expected from the next generation of experiments, some to be launched shortly, will also be presented.
\end{abstract}

\section{Historical Comments}

This conference was organized to allow us to focus on the character and origin of the ISM. It is especially concerned with the hot $\left(\sim 10^{6} \mathrm{~K}\right)$ and warm $\left(\sim 10^{3} \mathrm{~K}\right)$ components. A substantial amount of new data has been obtained since the last conference on this topic, including results from satellites including ROSAT, ULYSSES, HST, and EUVE, and from high-resolution ground-based optical studies. I will summarize some of the results on this topic obtained from the EUVE observatory, but first it may be worthwhile to pause and reflect upon some of the progress that we have made in this field. Let me start with reminders of a few highlights. I realize I will be slighting by omission almost everyone who has been working in this field, and I apologize for my limited choice of examples. However, a full recounting of all the effort that has been expended on this topic would overfill the Conference Proceedings all by itself.

It wasn't until the late 1960s that the hot component of the ISM was detected by Bowyer et al (1968), confirming an earlier prediction by Spitzer (1956). This initial observation showed the soft X-ray $1 / 4 \mathrm{keV}$ flux was anticorrelated with the Galactic hydrogen column, as derived from $21 \mathrm{~cm}$ radio observations, thereby demonstrating that at least the majority of the flux had something to do with our Galaxy. However, the flux scaled as if the absorbing ISM cross-section was one-third of its true value, which was a truly puzzling finding.

Ten years later a number of high-resolution spectroscopy observations of hot stars in the $900-1200 \AA$ band were carried out with the Copernicus satellite. These showed the O VI $1032 \AA$ line in absorption. A perplexing fact was that this line was narrow, which is best generated by a plasma at a temperature of a few $\times 10^{5}$ degrees. Hence the material producing this absorption seemed to be independent of the soft X-ray producing higher temperature gas. 
In the 1980s, a number of soft X-ray all-sky maps were obtained by Johns Hopkins (Davidsen et al, 1972), MIT (Marshall \& Clark, 1984), and Wisconsin (McCammon et al, 1983), and the Wisconsin group carried out a number of rocket flights which provided information on the flux in bands centered at roughly 90 and $120 \AA$ (Bloch et al, 1986). The $1 / 4 \mathrm{keV}$ maps provided exquisite detail on the anti-correlation of this flux with hydrogen column, and confirmed that its intensity varied with an ISM cross-section of approximately one-third of its expected value. The flux in the lowest energy bands did not track with hydrogen column, which added a new dimension to the puzzle.

A variety of theorists rose to the challenge of weaving the experimental data into a coherent whole, led perhaps by the hope that this would be a simple problem, easily solved. George Field and colleagues (1969) developed a theory with thermal pressure equilibrium between warm $\left(10^{4}\right.$ degree $)$ neutral clouds and hot material. This model was dealt a decisive blow by the discovery of O VI in the ISM. McKee and Ostriker (1977) developed an overall model of the ISM with warm cloud / hot gas interfaces, which provided among other things an elegant explanation of the observations of O VI.

The overall morphology of the local high temperature gas was explored in detail by the Wisconsin group in the 1970s and 1980s, who developed the displacement model as an explanation for the soft X-ray background (Cox \& Reynolds, 1987). This model reached its peak expression in the depiction of the local bubble. This term is now incorporated into the astronomical lexicon and is the title of our conference.

Jakobsen and Kahn (1986) revived the conceptual model of absorbing clouds interspersed in a hot medium, and showed that this model could also explain most of the data. While both the Jakobsen and Kahn model and the displacement model fit the observations reasonably well, each had its own set of shortcomings. Unfortunately, the available data was not sufficient to provide an overwhelming case for either model, though the interstellar clouds needed for the Jakobsen and Kahn model did not appear to be present.

Heiles and Kulkarni (1988) reviewed a substantial amount of observational material and concluded that the pervading warm neutral material is typically in sheets, rather than in clouds like those usually associated with the ISM.

The major theoretical advance of the 1990s, in my view, was the work of Breitschwerdt and Schmutzler (1994), who showed that the ionization state of the ISM was strongly dependent upon the thermal history of the material, and that in extreme cases the thermal temperature of the electrons could be vastly different from the photon distribution. Whether this concept is one that Nature has, in fact, provided, is yet to be determined.

In this sweeping overview, let me show a few illustrative examples of where we have been and where we are now. In Figure 1, I show one of the cartoons depicting the local bubble as developed by the Wisconsin group (Snowden et al, 1990). This bubble was hypothesized to have been produced 


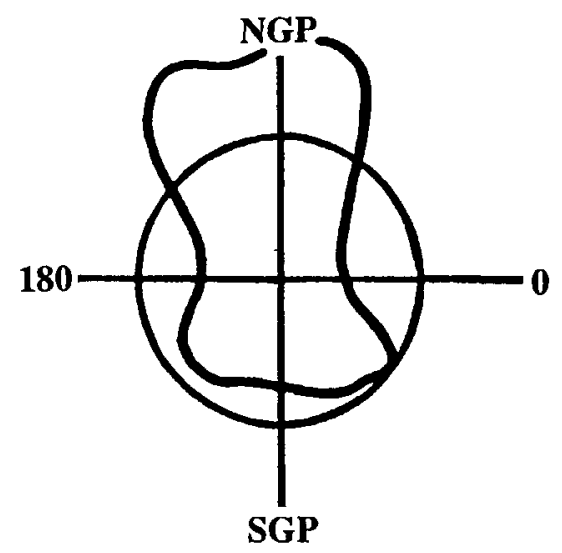

Fig. 1. A classic depiction of the "Local Bubble" as envisioned in the late 1980s. (From Snowden et al, 1990.)

by a supernova that occurred nearby which displaced the local material into a boundary. The morphology of the bubble was tuned to provide the different intensities between the poles and the plane of the Galaxy, which is roughly a two-to-one effect. Some emission was hypothesized to come from the hot material within the cavity, but since the emission measure in this cavity was not sufficient to produce all of the flux, some flux had to be produced at the boundary walls.

With the advent of ROSAT, a variety of new information has been obtained. Without doubt the most spectacular was the observation of soft X-ray shadows (Snowden et al, 1991; Burrows \& Mendenhall, 1991) which showed that the "local bubble" was not, in fact, local, but that much of the $1 / 4 \mathrm{keV}$ emission in the general direction of the Galactic poles was coming from distant regions. In principle, one can still invoke a wall boundary in the Galactic plane, (see, for example, Snowden, this volume) but the supporting data are quite thin and may be more easily explained in other ways.

Of substantial note is that an explanation has now been found for the curious behavior of the soft X-ray absorbing characteristics of the ISM. A number of investigations (Sidher et al, 1996, Snowden et al, 1997, several papers in this volume) have shown that several components contribute to the overall soft X-ray flux, including (among others) local emission, Galactic halo emission, and an extragalactic component. It is the summed effect of these components that produce a result that mimics a constant flux with an unnatural ISM absorbing cross section. 


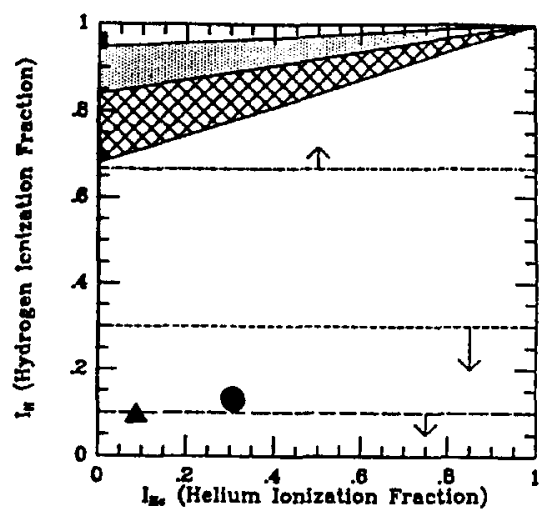

Fig. 2. The ionization state of the ISM as derived by several authors before the advent of spectroscopic data from EUV and HUT. (Results from several articles in the refereed literature whose authors need not be identified at this point.)

So much for a broad-brush overview. At this point I would like to summarize some of the work that has been carried out with the Extreme Ultraviolet Explorer, which provides us with new information on the character of the ISM.

\section{Recent Results from $E U V E$}

\subsection{The Ionization State of the Interstellar Medium}

The ionization state of the ISM was generously stated to be "uncertain" before the advent of EUVE observations. In Figure 2, I show a summary of most of the results available in the late 1980s. In general, authors attempted to derive a single, global ionization state of the ISM; these were obtained by rather indirect means. The wide variety of results obtained indicate the large degree of uncertainty regarding this parameter at that time.

With the advent of EUV spectroscopy, a substantial number of objects were observed which showed the effects of the intervening ISM. For studies of the ISM, the best choice of a background continuum source is a hot white dwarf. Many studies have been carried out with EUVE on hot white dwarfs, not only for the intrinsic information concerning the hot white dwarfs themselves, but also to obtain information on the ISM. The ISM H I and He I absorption in the spectra of six hot white dwarfs were derived from data obtained with EUVE. These data showed that the neutral helium to neutral hydrogen ratio was reasonably fit by a value 0.07 , which is substantially 


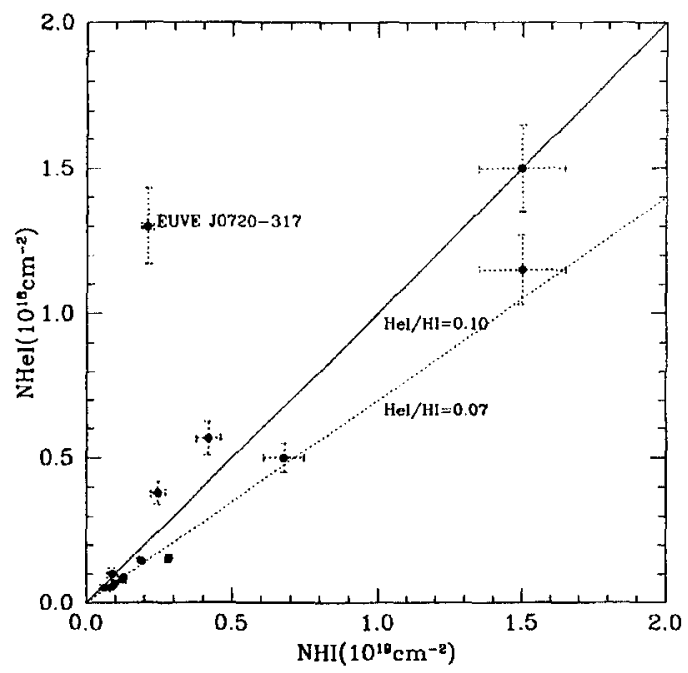

Fig. 3. Ratios of He I/H I for 12 stars within $200 \mathrm{pc}$.

less than the cosmic abundance of helium to hydrogen of 0.1 (Dupuis et al, 1995). This suggests that some of the ISM helium is ionized. There was also a suggestion of a variation in this parameter with differing look directions, but this effect was not strongly supported by the data.

The results of a study of ISM absorption to more distant stars are shown in Figure 3 where the data are combined with the earlier results cited above (Dupuis et al, in preparation). The data on the more distant stars, because of their relative faintness, have larger error bars than the data on the nearby stars; nonetheless, this extended data set clearly indicates that there are substantial variations in the ionization state of the ISM in different view directions.

We can use the He II absorption edge at $228 \AA$ to obtain the fraction of singly ionized helium in the ISM. This requires a careful fitting of the absorption edge because of the auto-ionization effect present in the ISM, as described by Rumph et al (1994). With this information, we can then determine the total amount of ionized helium, since Heiles et al (1996) have shown that essentially no He III exists in the Galaxy. The sum of the neutral and ionized helium gives us the total helium in the line of sight, and with the assumption that the total hydrogen to total helium ratio is 10 , we can obtain the total amount of hydrogen in this line of sight. A comparison of this number with the column of neutral hydrogen in this line of sight yields the column of H II, giving us a complete picture of the ionization state of the ISM. 
Table 1. Percentage Ionized $\mathrm{H}$ and $\mathrm{He}$ for Four View Directions

\begin{tabular}{ccc}
\hline Star & \multicolumn{1}{c}{ Ionized H lonized He } \\
\hline HZ43 & $<40$ & $<50$ \\
GD153 & $<50$ & $<60$ \\
EUVE J0720-317 & $\sim 88$ & $\sim 28$ \\
EUVE J0723-277 & $\sim 65$ & $\sim 62$
\end{tabular}

I show in Table 1 results obtained in the direction of four stars. These examples are extreme cases which emphasize the fact that the ionization state of the ISM can vary substantially in different view directions. More work on this topic is in progress.

\subsection{Is There Thermal Pressure Equilibrium Between the Warm and Hot Components of the ISM?}

Almost all models of the warm and hot phases of the ISM assume thermal pressure equilibrium between these two phases, beginning with the original suggestion of Spitzer, and continuing with the two phase model of the ISM by Field and co-workers, and the McKee and Ostriker model. Other models have been suggested which invoke magnetic fields as one of the components providing pressure balance, but these have not achieved wide currency.

What is the evidence in favor of a thermal pressure balance? A wide variety of values have been obtained for the thermal pressure of the hot phase of the ISM. A number of these values have been obtained by measuring the thermal pressure of cool clouds and then assuming that the hot gas is at the same pressure. This assumes thermal pressure equilibrium rather than establishes thermal pressure equilibrium.

The best direct estimates of the thermal pressure of the hot gas come from soft X-ray measurements and EUV measurements (Bowyer et al. 1997). A problem with soft X-ray measurements is that the penetrating power of soft $\mathrm{X}$-rays is relatively large, and as a consequence the flux seen by an observer may be some combination of flux in the line of sight to a cloud, plus flux from beyond the cloud that has been partially attenuated by the cloud. Or, alternatively, a cloud may be so dense that it is totally opaque to the soft Xrays, but the flux observed is partially attenuated by a smaller inconspicuous cloud in the line of sight to the totally obscuring cloud. This situation is demonstrated in Figure 4. In Figure $4 \mathrm{I}$ also show the situation for EUV emission. EUV radiation is sufficiently soft that it is absorbed by even small clouds in the local ISM, and one can be certain that the flux is not coming from regions behind the cloud.

We have measured the EUV flux in the direction of a shadow cast by a cloud in the overall background, and have investigated the absorbing material 


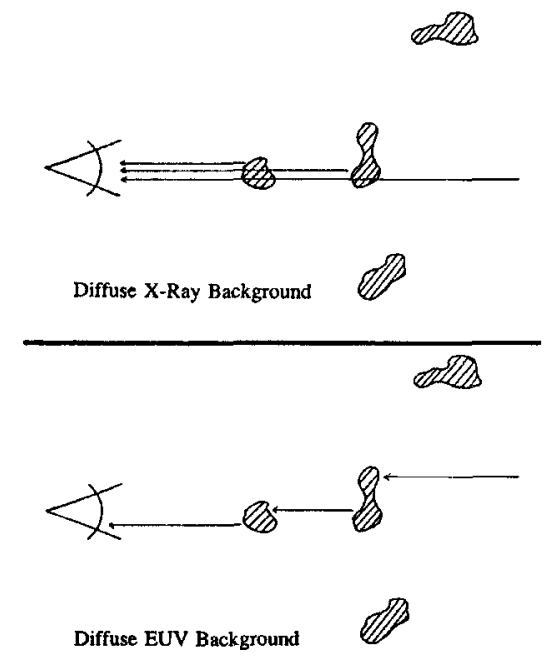

Fig. 4. A cartoon depicting the intrinsic difference between the measurement of flux in the soft X-rays and the EUV. The relatively large penetrating power of soft $\mathrm{X}$-rays renders it difficult, if not impossible, to identify flux which is not the product of several emitting columns.

in the line of sight to this cloud using optical techniques (Bowyer et al. 1997). These data showed that the EUV flux measured directly in the line of sight to this absorbing cloud (at a distance of $\leq 40 \mathrm{pc}$ ) is due to unabsorbed emission between us and the cloud. A pressure of $\mathrm{P} / \mathrm{K} \sim 19,000$ was obtained for the hot component of the ISM in this direction (Bowyer et al, 1995). This pressure can be compared with the pressure of the local cloud surrounding the Sun, which has been independently determined by several techniques. The canonical value for the pressure of the local cloud is $\mathrm{P} / \mathrm{K} \sim 700$; a more recent determination obtained by Vallerga (1996) suggests $\mathrm{P} / \mathrm{K} \sim 2000$. A comparison of the pressure of the hot component with either of these two numbers shows that there is not thermal pressure equilibrium between the local cloud and the nearby hot ISM at least in this direction.

An escape which will allow us to retain the concept of thermal pressure equilibrium is provided by the fact that the pressure will certainly be different as an interstellar shock wave passes through the region. Although there is no evidence for a local shock within 40 parsecs of the Sun, this is still a hypothetical possibility. A second potential escape from this dilemma is provided by the fact that the zero-level calibration for the EUVE data employed in this study was not definitively established, and by assuming a gross miscalculation of this zero level, a lower pressure for the hot ISM could be obtained. 
New EUVE measurements have been obtained for two more clouds producing shadows in the EUV background. These are at widely different lines of sight. An analysis of these data (Berghöfer et al, this volume) incorporates a new approach in determining the pressure. A differential measurement is made by comparing the flux from a nearby cloud with that from a more distant cloud, thereby giving a pressure measurement for that segment of the emitting region. When these results are extrapolated to zero distance, one obtains an estimate of the true zero flux level for the detector. This technique has been applied to the data from the original cloud shadow and to the two new shadows.

The surprising result is that the pressure in all three of these disparate view directions is the same, about $\mathrm{P} / \mathrm{K} \sim 16,000$, and the extrapolation of the results to zero distance for each of these measurements gives the same zero level flux. Using this zero level, one can make a revised estimate of the nearby pressure in the direct line of sight to the nearby clouds. Again, the pressures to the nearby clouds are the same in all three directions, and they are the same as the pressures obtained for the segments between the nearby clouds and the more distant clouds. It seems extraordinarily implausible that all of these results could give the same answer by chance. We conclude we have obtained a true measurement of the pressure in our local region.

We also note that the option of a different pressure between us and a nearby cloud which could be produced by a passing shock wave is no longer viable because of the disparate view directions sampled. We conclude that thermal pressure equilibrium between the local warm cloud and the hot component of the ISM does not exist, and hence this cloud, and by implication, other clouds, must be constrained by some other mechanism than thermal pressure.

\subsection{Future Prospects}

The majority of our knowledge of the hot component of the ISM has come from studies of the morphological characteristics of the soft X-ray background. Several papers in this conference have utilized the broad energy discrimination capabilities of the ROSAT proportional counters to obtain new insights as to the character and distribution of the emitting material, foreshadowing the potential of higher resolution spectral studies. Spectroscopy of the diffuse soft X-ray and EUV background is intrinsically quite difficult and is rooted in the fact that the intensity of the radiation from any small region is quite small. One is forced to a trade between instruments which have large flux-collecting capabilities and lower energy resolution capabilities, such as proportional counters, CCDs, and microcalorimeters, and classic slit-grating combinations which have higher spectral resolution but low flux collecting capabilities. 
Table 2. Instruments to Study the Diffuse Soft X-Ray / EUV Background

\begin{tabular}{lcc}
\hline Experiment & $\begin{array}{c}\text { Bandpass } \\
(\AA)\end{array}$ & $\begin{array}{c}\text { Resolution } \\
(E / \Delta E)\end{array}$ \\
\hline ROSAT (Pfeffermann et al. 1986) & $44-100$ & 5 \\
(lowest energy channel) & & \\
EUVE (Jelinsky et al. 1995) & $190-250$ & 10 \\
& $400-460$ & 10 \\
Alexis (Bloch \& Smith 1991) & $130-190$ & 10 \\
DXS (Sanders \& Edgar 1996) & $40-80$ & 20 \\
$C U B I C$ (Burrows et al. 1995) & $10-50$ & $40-60$ \\
UCB/EURD & $350-1100$ & $70-200$ \\
\hline
\end{tabular}

I show in Table 2 the energy resolution capabilities of a variety of instruments which have been used to study the diffuse X-ray background, and some that are expected to be deployed in the future.

The ROSAT PSPC energy resolution (Pfeffermann et al, 1986) is such that the majority of the work performed with this instrument has involved morphological studies. These have been quite productive. However, it is not clear that more data of this type will be especially useful.

Jelinsky et al (1995) used the EUVE satellite to look for lines expected to be present in the hot ISM. Although the energy resolution was quite low (the instrument was not designed to study diffuse objects, but was designed to study point sources), it was nevertheless found that several lines were not present which should have been detected if the ISM were in collisional ionization equilibrium. A possible solution that was suggested was that depletion had taken place in the ISM, but a factor of 10 depletion was necessary for the results to be compatible with the upper limits obtained. Recent observations by Fitzpatrick (1996) have shown that this depletion is typically only a factor of 3 for most elements. This is certainly a strong suggestion that something in our assumptions about the character of the ISM is incorrect.

The Alexis instrumentation (Bloch \& Smith, 1991) has not yet produced useful results pertaining to the ISM, and it now appears unlikely that it will be capable of doing so. The DXS instrument (Sanders \& Edgar 1996) has somewhat higher resolution than Alexis, but the data produced are difficult to interpret. If one assumes that the instrument functioned correctly, the most that one can say is that line emission is present, and that higher energy resolution will certainly be required to obtain useful information on the ISM.

The CUBIC instrument (Burrows et al, 1995) has substantially higher spectral resolution and a large grasp. Most of its response is at higher energies, where the extragalactic diffuse X-ray background overwhelms the local ISM component. But sufficient capabilities exist at lower energies that this instrument, if successfully flown, will surely add to our knowledge of the ISM. 
We have developed a slit spectrometer for the study of diffuse EUV radiation which provides much higher spectral resolution, but with a corresponding loss in grasp. However, the grasp of the instrument is almost an order of magnitude larger than that of a classic slit spectrometer. This improvement has been obtained by employing a new grating which is especially designed for the study of diffuse flux. This instrument will be capable of detecting individual lines from the thermal plasma producing the soft X-ray / EUV emission with reasonable integrations. Long integration times are feasible with this instrument because of a variety of techniques which have been employed to reduce the instrumental background to extremely low levels.

We have fabricated and calibrated three of these instruments (Bowyer et al, 1997; Morales et al, this volume). All are very similar; the most substantial difference is that one of the three employs a slightly different grating tilt that emphasizes shorter EUV wavelengths. This spectrograph employs different coatings to enhance the shorter wavelength response.

One of these spectrometers (the Ultraviolet Cosmic Background, $U C B$ ) (Bowyer et al. 1997), covering the 500-1100 $\AA$ band, is part of the payload of NASA's Small Spacecraft Technology Initiative satellite, Lewis. It is scheduled for launch in the second half of 1997. Two of these spectrometers, one covering the 500-1100 $\AA$ band and the other covering the 350-800 $\AA$ band, constitute the instrument Espectrografo Ultravioleta extremo para la observacion de la Radiacion Difusa ( $E U R D$ ). It is described in some detail in the paper by Morales et al (these proceedings). EURD is part of the instrument complement of the Spanish MINISAT 01 satellite. EURD is a collaboration of the Spanish Instituto Nacional de Technica Aeroespacial (INTA) and the Center for EUV Astrophysics, University of California at Berkeley. The reduction and analysis of $E U R D$ data will be carried out as a collaboration between the University of California at Berkeley and an INTA team lead by C. Morales. The MINISAT 01 satellite was successfully launched in April 1997.

An example of the lines expected in the EUV and the sensitivity of these instruments are shown in Figure 5, along with current upper limits to the diffuse EUV background. The resolution of these instruments is 4-5 $\AA$; this is smaller than the line widths shown in the figure.

\section{Coda}

Several speakers have commented on open questions regarding the character of the soft X-ray / EUV flux produced in our Galaxy. In the following, I provide my own personal list of questions which $I$ hope will be answered in the foreseeable future.

- Is the local bubble a "Local Bubble" or a generalized region produced by multiple events? 


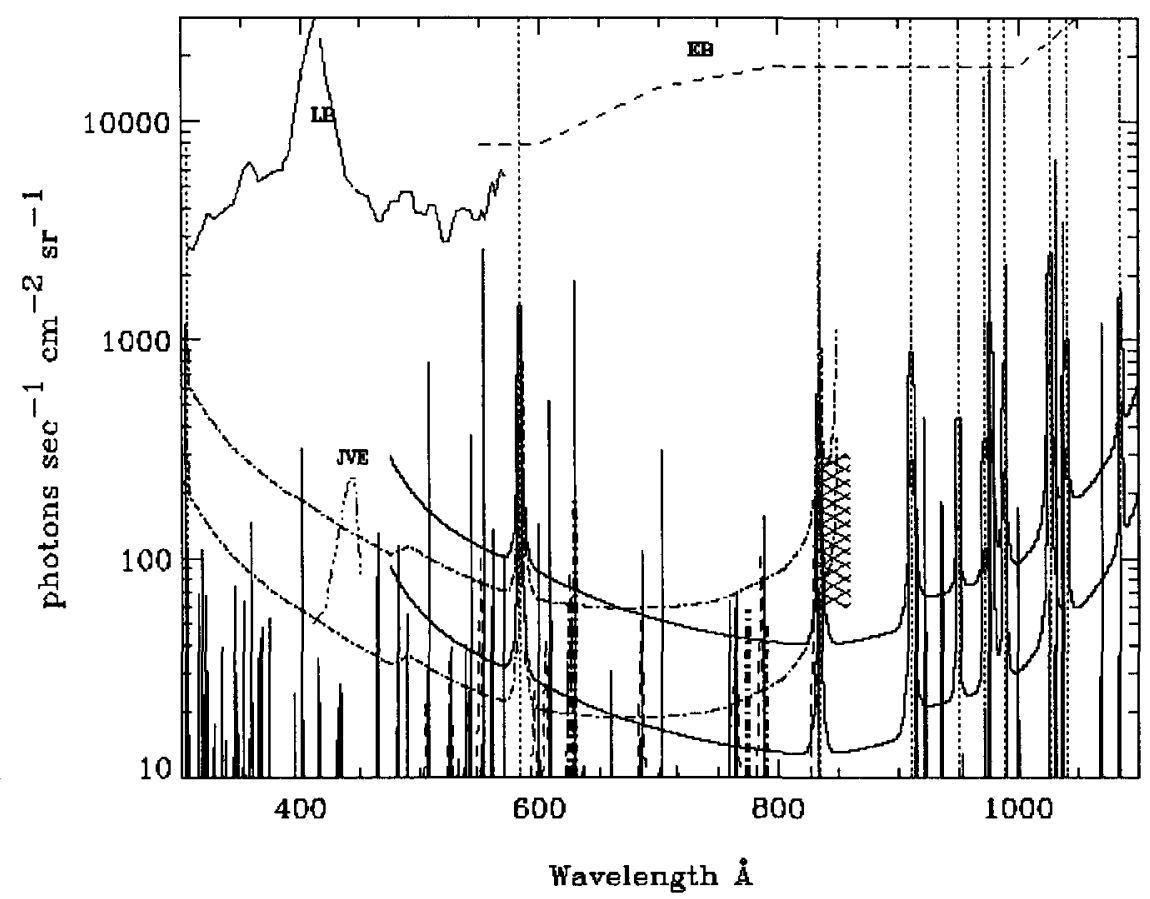

Fig. 5. The minimum measurable flux for the $U C B$ and $E U R D$ spectrometers and existing upper limits to the diffuse EUV cosmic background. The curve marked LB is the $15 \AA$ resolution upper limit of Labov and Bowyer (1991). The curve marked $\mathrm{EV}$ is the $30 \AA$ resolution upper limit derived from Voyager data by Edelstein, Bowyer, and Lampton (1997). The curve marked JVE is the upper limit of Jelinsky et al (1995) using EUVE data. The curved solid lines are the flux limits provided by 200 and $1000 \mathrm{hr}$. of observation with the long wavelength $U C B$ spectrometer. The dash-dotted curves are the corresponding limits for the short wavelength $E U R D$ spectrometer. The solid vertical lines are expected ISM emission lines from a steady-state collisionally ionized plasma. The heavy vertical bars are the intensities from the delayed recombination model of Breitschwerdt and Schmutzler (1994) and Breitschwerdt (1997, private communication). The dotted vertical lines are the expected nighttime airglow. The cross-hatched region shows the range of the emission signature predicted by Sciama (1994) for a Galactic halo of radiatively decaying neutrinos.

- Is the plasma in collisional ionization equilibrium, or is it in substantial or even extreme non-equilibrium? What are the "temperatures" of the components?

- Are clouds or sheets the primary structures for the warm neutral component?

- Are "clouds" pressure or magnetically confined? 
Acknowledgements. I wish to thank the Conference Organizers for devising this timely meeting and for carrying it through with such elegance.

\section{References}

Bloch J., Jahoda K., Juda M., McCammon D., Sanders W. \& Snowden S. (1986): ApJ Lett, 308, L59

Bloch J. \& Smith B. (1991): in Extreme Ultraviolet Astronomy, ed. S. Bowyer and R. Malina (New York: Pergamon), 289

Bowyer S., Field G. \& Mack J. (1968): Nature 217, 32

Bowyer S., Lieu R., Sidher S. D., Lampton M. \& Knude J. (1995): Nature, 375, 212

Bowyer S., Edelstein J. \& Lampton M. (1997): ApJ 485

Breitschwerdt D. \& Schmutzler T. (1994): Nature 371, 774

Burrows D. \& Mendenhall J. (1991): Nature 351, 629

Burrows D. et al.(1995): Proc. SPIE, 2518, 141

Cox D. \& Reynolds R. (1987): ARA\&A 25, 303

Davidsen A., Shulman J., Fritz G., Meekins J., Henry R. \& Friedman H. (1972): ApJ 177, 629

Dupuis J., Vennes S., Bowyer S., Pradhan A. \& Thejll P. (1995): ApJ 455, 574

Dupuis J., Vennes S. \& Bowyer S. (1997): in preparation

Edelstein J., Bowyer S. \& Lampton, M. (1997): submitted to ApJ

Field G., Goldsmith D. \& Habing H. (1969): ApJ Lett 155, L49

Fitzpatrick E. (1996): ApJ Lett 473, L55

Heiles C. \& Kulkarni S. (1988): in Galactic and Extragalactic Radio Astronomy,

Second Edition, ed. Verschuur G., Kellermann K., Springer

Heiles C., Coo B.-C., Levenson N. \& Reach W. (1996): ApJ 462, 326

Jakobsen P. \& Kahn S. (1986): ApJ 309, 682

Jelinsky P., Vallerga J. \& Edelstein J. (1995): ApJ 442, 653

Labov S. \& Bowyer S. (1991): ApJ 371, 810

Marshall F. \& Clark G. (1984): ApJ 287, 633

McCammon D., Burrows D., Sanders W. \& Kraushaar W. (1983): ApJ 269, 107

McKee C. \& Ostriker J. (1977): ApJ 218, 148

Pfeffermann E. et al.(1986): Proc. SPIE 733, 519

Rumph T., Bowyer S. \& Vennes S. (1994): AJ 107, 2108

Sanders W. \& Edgar R. (1996): in Astrophysics in the EUV, ed. S. Bowyer and R. Malina (Dordrecht: Kluwer), 269

Sciama D. (1994): Modern Cosmology and the Dark Matter Problem (New York: Cambridge University Press)

Sidher S., Sumner T., Quenby J. \& Gambhir M. (1996): A\&A 305, 308

Slavin J. \& Cox D. (1992): ApJ 392, 131

Snowden S., Cox D., McCammon D. \& Sanders W. (1990): ApJ 354, 211

Snowden S., Mebold U., Hirth W., Herbstmeier U. \& Schmitt J. (1991): Science 252,1529

Snowden S., Egger R., Freyberg M., McCammon D., Plucinsky P., Sanders W., Schmitt J., Trümper J. \& Voges W. (1997): ApJ 485, 125

Spitzer L. (1956): ApJ 124, 20

Vallerga J. (1996): Space Sci. Rev. 78, 277 